\title{
Monitoring the Succinate Dehydrogenase Activity Isolated from Mitochondria by Surface Enhanced Raman Scattering ${ }^{\dagger}$
}

\author{
Katherine A. Hollywood, Iqbal T. Shadi, and Royston Goodacre* \\ School of Chemistry, Manchester Interdisciplinary Biocentre, University of Manchester, 131 Princess St, \\ Manchester M1 7DN, United Kingdom \\ Received: September 16, 2009; Revised Manuscript Received: December 18, 2009
}

\begin{abstract}
Monitoring enzyme kinetics is an important aspect of biochemistry and is essential when studying metabolism. In this study we demonstrate that succinate dehydrogenase activity of mitochondria can be analyzed quantitatively by surface enhanced Raman scattering (SERS). We used the artificial electron acceptor reporter molecule 2,6-dichlorophenolindophenol (DCPIP) that when oxidized is SERS active. On reduction this redox dye changes from blue to colorless and is SERS inactive. This color change allows UV/vis spectrophotometry to be used as a standard reference measurement as well as SERS. SERS analysis incorporated kinetic time course measurements and employed a portable laser Raman spectrometer using an excitation wavelength of $785 \mathrm{~nm}$ in conjunction with gold colloids. Good correlation coefficients and quantitative data were observed with the additional advantage that analysis by SERS also provided good fingerprint spectral information from vibrational band frequencies thus allowing the potential of multiplexing enzyme reactions. We believe that this is the first demonstration of monitoring succinate dehydrogenase activity with SERS and that SERS has considerable potential for being applied to the analysis of metabolic processes.
\end{abstract}

\section{Introduction}

The analysis of metabolic processes is important in all areas of biochemistry and particular interest is shown in the analysis of succinate dehydrogenase, the enzyme involved in the electron transfer reactions in all kingdoms of life as documented by refs $1-4$.

Succinate dehydrogenase is responsible for catalyzing the oxidation of succinate to fumarate within the citrate acid cycle (Figures 1C and 2A). In higher organisms all enzymes within the cycle are located within the mitochondrial matrix; however an exception to this is succinate dehydrogenase and its coenzyme flavin adenine dinucleotide (FAD). The enzyme complex (often represented by E-FAD) is tightly bound to the inner membrane of the mitochondrion allowing its easy isolation.

Upon isolation of this E-FAD it is possible to measure the succinate to fumarate reaction by monitoring the reduction of an artificial electron acceptor; for example, the Hill reagent 2,6dichlorophenolindophenol (DCPIP). When this redox dye is oxidized it is blue and when reduced it is colorless (see Figure $1 \mathrm{~A}$ and $\mathrm{B}$ for reactions), thus simple spectrophotometry can be employed to follow this redox change. In order to use this artificial electron acceptor, the normal path of electrons in the electron transport chain must be blocked. ${ }^{5}$ This is accomplished by adding sodium azide to the reaction mixture (Figure 2B). This poison inhibits the transfer of electrons from Complex IV (cytochrome $\mathrm{a}_{3}$ ) to the final acceptor, oxygen, so that the electrons cannot be passed along the preceding cytochromes and Ubiquinone (also called coenzyme Q). Instead these electrons are picked up by DCPIP. The reduction of DCPIP by E-FADH ${ }_{2}$ can then be measured at $600 \mathrm{~nm}$ (Figure 1B) through the observed blue to colorless color change. ${ }^{5}$

\footnotetext{
† Part of the "Martin Moskovits Festschrift".

* To whom correspondence should be addressed. Tel: +44 (0) 161 3064480. E-mail: roy.goodacre@manchester.ac.uk.
}

However, one of the problems with spectrophotometry is that no chemical information on the reporter is available which means that the method potentially lacks specificity. By contrast, Raman spectroscopy yields information rich spectral fingerprints due to specific vibrational band frequencies being probed from the molecules under interrogation. ${ }^{6}$ Unfortunately, this inelastic scattering of light is usually very weak (typically 1 in $10^{6}-10^{8}$ photons), and Raman spectra can often be difficult to obtain from organic molecules because of the presence of fluorescence, which is further compounded in biological matrices. These shortcomings in Raman spectroscopy can be largely overcome by surface enhanced Raman scattering $\left(\mathrm{SERS}^{7}\right)$, including both fluorescence ${ }^{8}$ and signal enhancement, which for certain molecules allows single molecule detection. ${ }^{9,10}$ Thus SERS has found wide utility as a sensitive analytical and bioanalytical technique. ${ }^{11-14}$

Recently there has been interest in applying SERS to monitor kinetics of reactions particularly those concerned with metabolism. Success in monitoring kinetics of synthetic chemical reactions using SERRS (a variant of SERS which includes a resonance enhancement) has been reported in, ${ }^{15}$ as well as those detailing general enzyme activity. ${ }^{16,17}$ Therefore the aim of the present study was to investigate whether SERS could be used to gain quantitative enzyme information from succinate dehydrogenase isolated from mitochondria.

\section{Experimental Section}

2.1. Chemical and Reagents. All chemicals and reagents were purchased from Sigma (D-mannitol, $\mathrm{KOH}$, sodium azide, sodium succinate, malonic acid, $\mathrm{MgCl}_{2} \cdot 6 \mathrm{H}_{2} \mathrm{O}$, poly(L-lysine) hydrobromide, gold tetra chloride, and trisodium citrate) with the exception of DCPIP (Acros), $\mathrm{KH}_{2} \mathrm{PO}_{4}, \mathrm{~K}_{2} \mathrm{HPO}_{4}$, (BDH), and hydrochloric acid (Fisher). All chemicals were used without further purification. Double deionised water was used for all experiments. 
A<smiles>O=C1C(Cl)=CC(=Nc2ccc(O)cc2)C=C1Cl</smiles>

B

$\mathrm{FADH}_{2}+\mathrm{DCPIP}_{\text {ox }}$ (Blue) $\longrightarrow \quad \mathrm{E}-\mathrm{FAD}+\mathrm{DCPIP}$ red (Colourless)

C

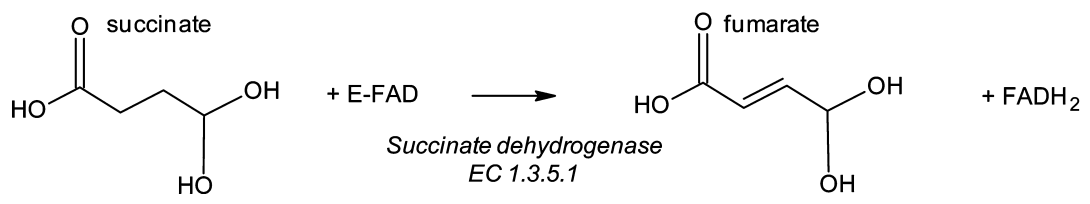

Figure 1. Detection of enzyme activity and reactions involved. (A) Schematic drawing of DCPIP (the final electron acceptor) in its oxidized and reduced form. (B) Representation of the reduction of DCPIP by $\mathrm{FADH}_{2}$ from its oxidized blue form of the dye to the reduced colorless form. (C) The reaction catalyzed by the enzyme succinate dehydrogenase (EC 1.3.5.1).

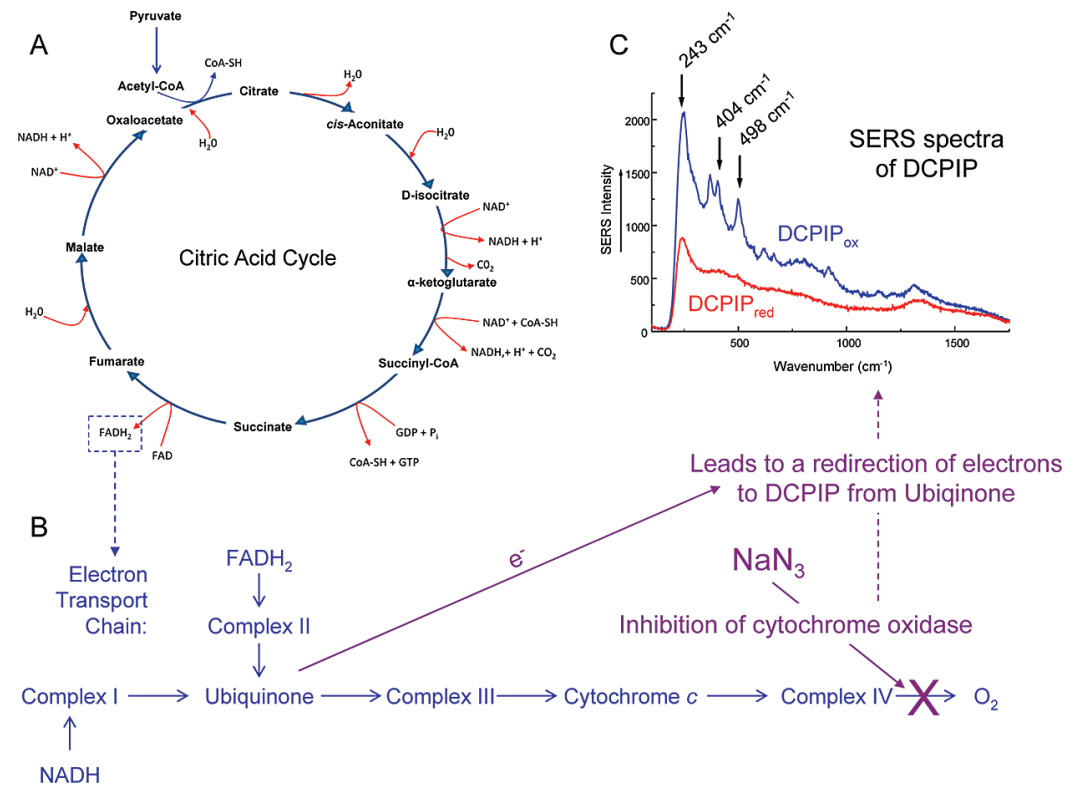

Figure 2. Schematic representations of (A) the citric acid cycle showing where succinate is converted into fumarate and (B) the electron transport chain detailing where sodium azide inhibits cytochrome oxidase. (C) SERS spectra of the artificial electron acceptor DCPIP in its oxidized and reduced forms which occurs due to the redirection of electrons from the electron transport chain.

2.2. Extraction of Mitochondria. Cauliflowers were obtained from a national retail outlet. $20 \mathrm{~g}$ from the $2-3 \mathrm{~mm}$ of the surface of cauliflower florets was added to $40 \mathrm{mLs}$ of ice cold grinding media $\left(0.3 \mathrm{~mol} \mathrm{dm}{ }^{-3}\right.$ mannitol, $0.006 \mathrm{~mol} \mathrm{dm}^{-3}$ $\mathrm{KH}_{2} \mathrm{PO}_{4}, 0.014 \mathrm{~mol} \mathrm{dm}{ }^{-3} \mathrm{~K}_{2} \mathrm{HPO}_{4}$, adjusted to $\mathrm{pH} 7.2$ ) and blended in a Ferretti FEBL10 food processor for $5 \mathrm{~min}$. The ground material was filtered through a muslin cloth and the supernatant transferred into chilled centrifuge tubes and centrifuged (Sorvall RC-5B refrigerated superspeed centrifuge) at $1778 \mathrm{~g}$ for $10 \mathrm{~min}$. The postnuclear supernatant was decanted into clean chilled centrifuge tubes and centrifuged (Sorvall RC$5 \mathrm{~B}$ refrigerated superspeed centrifuge) at $23042 \mathrm{~g}$ for $30 \mathrm{~min}$. The mitochondria pellet was recovered and resuspended in manitol assay medium $\left(0.3 \mathrm{~mol} \mathrm{dm}^{-3}\right.$ mannitol, $0.006 \mathrm{~mol} \mathrm{dm}^{-3}$ $\mathrm{KH}_{2} \mathrm{PO}_{4}, 0.014 \mathrm{~mol} \mathrm{dm}^{-3} \mathrm{~K}_{2} \mathrm{HPO}_{4}$, and $0.005 \mathrm{~mol} \mathrm{dm}^{-3} \mathrm{MgCl}_{2}$, adjusted to $\mathrm{pH}$ 7.2).

2.3. Monitoring Enzyme Activity. In a test tube, $0.5 \mathrm{~mL}$ of sodium azide $\left(0.04 \mathrm{~mol} \mathrm{dm}^{-3}\right), 0.5 \mathrm{~mL}\left(5 \times 10^{-4} \mathrm{~mol} \mathrm{dm}^{-3}\right)$ of DCPIP, $0.5 \mathrm{~mL}$ of succinate $\left(0.02 \mathrm{~mol} \mathrm{dm}^{-3}\right)$, and $0.9 \mathrm{~mL}$ of mitochondrial suspension was added to $3.2 \mathrm{~mL}$ of assay media and mixed. The mixture was allowed to equilibrate for 5 min after mitochondria addition. The kinetics of the reaction was subsequently measured over a period of 5-30 min using a Thermo-2 UV-vis spectrophotometer set at $600 \mathrm{~nm}$ as well as by SERS.

For SERS measurements an aliquot of $150 \mu \mathrm{L}$ of the above mixture was taken at 5 min intervals and added immediately to the gold SERS colloid (vide infra) with subsequent collection of SERS spectra.

In addition to the above reaction mixture, controls were also incorporated into the analysis and included:

-Removing only the sodium azide inhibitor. This is the negative control so that the electron transport chain functions normally and the terminal electron acceptor is oxygen (Figure 2B). 


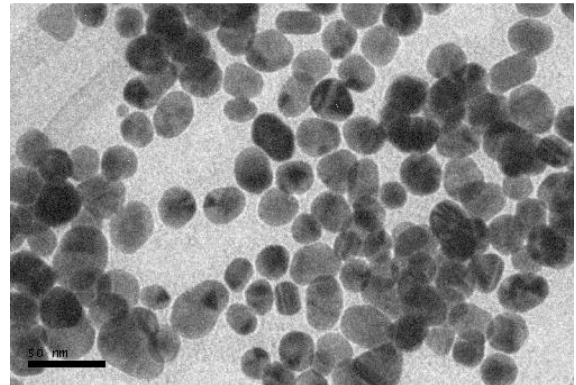

Figure 3. Transmission electron micrograph of citrate reduced $\mathrm{Au}$ colloids showing good homogeneity. The scale bar is $50 \mathrm{~nm}$.

- Removing only the sodium succinate. This is another negative control where as there is no substrate no electron transport will occur.

-Adding $2 \mathrm{~mL}$ of malonic acid $\left(0.2 \mathrm{~mol} \mathrm{dm}^{-3}\right)$. This was included as malonic acid is a competitive inhibitor for succinate dehydrogenase.

-Reducing the volume of the mitochondria preparation to 0.3 or $0.6 \mathrm{~mL}$.

2.4. Surface Enhanced Raman Scattering. 2.4.1. Colloid Preparation. A gold colloid was prepared as detailed in. ${ }^{10}$ All glassware were acid washed with aqua regia $\left[\mathrm{HNO}_{3} / \mathrm{HCl}(1: 3\right.$ $v / v)$ ] followed by gentle scrubbing with a soap solution. Colloid was prepared by reduction of $\mathrm{HAuCl}_{4}$ by sodium citrate. Briefly, $250 \mathrm{~mL}$ of $1 \mathrm{mM}$ solution of $\mathrm{HAuCl}_{4}$ was brought to the boil followed by addition of $25 \mathrm{~mL}$ of $38.8 \mathrm{mM}$ solution of sodium citrate. The mixture was boiled for $30 \mathrm{~min}$ followed by cooling at room temperature. The quality of the resulting colloid was checked by determining the wavelength of the absorption maximum in the visible region on a Thermo-2 UV-vis spectrophotometer. The nature of the colloid often used for SERS, has been examined using visible absorption, photon correlation and NMR spectroscopic techniques (data not shown) which confirm that the surface of the gold particles are covered with a layer of citrate with pendent negatively charged groups. However, the subsequent addition of poly(L-lysine) which coats the surface, results in the presence of pendent positively charged groups on the colloidal surface. ${ }^{18}$

The citrate-reduced sols gave an absorption maximum at $\sim 529 \mathrm{~nm}$. An acceptable gold sol is one which not only absorbed at $529 \mathrm{~nm}$ but has an absorption band at this wavelength with a half height width of $\sim 50 \mathrm{~nm}$, and this was the case for this preparation (data not shown). TEM of the gold sols (Figure 3) showed that the particles had a mean diameter of $20 \mathrm{~nm}$ and had good uniformity (Figure 3).

2.4.2. Aggregation. In initial experiments the aggregation of the citrate reduced gold colloid particles was induced by the addition of poly(L-lysine) as this has been found by use to be very important for SERS of dye molecules. ${ }^{8}$ In this process $150 \mu \mathrm{L}$ of a $0.01 \%$ aqueous solution of poly(L -lysine) was added to $1 \mathrm{~mL}$ of gold colloid, which had been diluted with 1 $\mathrm{mL}$ of deionised water, followed by $150 \mu \mathrm{L}$ of the dye solution. The $\mathrm{pH}$ was then adjusted to enhance SERS ${ }^{19}$ by the addition of $35 \mu \mathrm{L}$ of hydrochloric acid, $1 \mathrm{~mol} \mathrm{dm}{ }^{-3}$ of $\mathrm{KOH}$, or $10 \mu \mathrm{L}$ of $1 \mathrm{~mol} \mathrm{dm}{ }^{-3} \mathrm{KOH}$.

The above was found to give very little SERS (see below) so for subsequent experiments poly(L-lysine) was excluded with the rest of the analysis mixture as detailed above. A phenomenon that has been previously observed. ${ }^{20}$ For the enzyme reactions the $150 \mu \mathrm{L}$ of the dye solution was replaced with $150 \mu \mathrm{L}$ of the enzyme reaction.

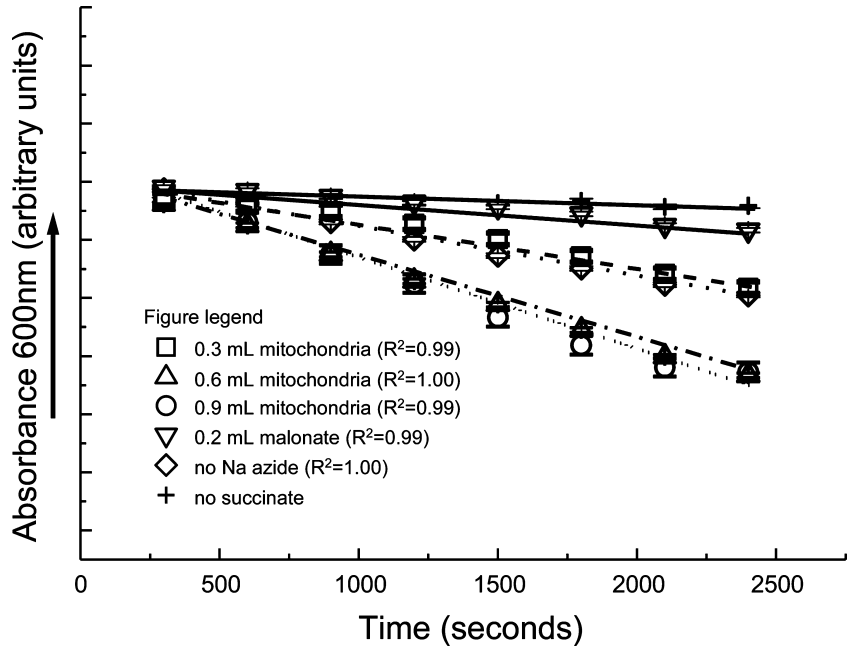

Figure 4. Graph representing the kinetic experiments (DCPIPox $\rightarrow$ DCPIPred) monitored spectrophotometrically. The wavelength used for analysis is $600 \mathrm{~nm}$. From the top to bottom are the controls employed to assay the enzyme activity; see text for full details. Correlations coeffients $\left(R^{2}\right)$ are also shown in the inset.

2.4.3. Instrumentation. For Raman analysis spectra were collected using an Ahura Defender system (Ahura Scientifics, Wilmington, MA), a hand-held, portable Raman spectrometer (using back scattered geometry) with a built in $4 \mathrm{~mL}$ sample chamber which provided at the sample $300 \mathrm{~mW}$ laser power at $785 \mathrm{~nm} \pm 0.5 \mathrm{~nm}$ laser excitation and $2 \mathrm{~cm}^{-1}$ line width. The spectrometer has a monochromatic spectral range of 781-1014 $\mathrm{nm}$, a Raman spectrum range of $250-2875 \mathrm{~cm}^{-1}$ and a spectral resolution of $7-10.5 \mathrm{~cm}^{-1}$ (full width at half-maximum; fwhm). Built into the Ahura spectrometer are Rayleigh rejection filters of OD 7, a silicon CCD 2048 pixels detector in direct dispersive detection mode and a dispersion mode which is a single pass spectrometer (1200 grooves/mm blazed at $900 \mathrm{~nm}$ ).

The SERS colloid-analyte aggregate was analyzed by placing the sample into the $4 \mathrm{~mL}$ sample chamber. All samples were analyzed three times to give three machine replicate measurements.

\section{Results and Discussion}

3.1. Validation of the Enzyme Systems under Study. Before any SERS analyses took place, it was important to make sure that the succinate dehydrogenase assay using an artificial electron acceptor with visible spectrophotometry as detailed in Figures 1 and 2 was functioning. Therefore as detailed above a series of positive and negative controls were conducted. The positive controls included varying the volume of the mitochondria preparation used $(0.3,0.6$, or $0.9 \mathrm{~mL})$, while the negative controls included not having any succinate substrate present or any sodium azide inhibitor. In addition, malonic acid was added with succinic acid in the reaction mixture as malonate is a competitive inhibitor for succinate dehydrogenase.

The results of these preliminary experiments are shown in Figure 4 where the absorbance of DCPIP at $600 \mathrm{~nm}$ is plotted against reaction time. It can be seen that there was no enzyme reaction measured in the negative control that contained no succinic acid substrate (symbols = pluses) as the slope of the time reaction is more or less flat. This slope was also very close to the reaction that contained malonic acid which inhibited the succinate dehydrogenase enzyme (downward pointing triangles). The reaction mixture containing no sodium azide (diamonds) still allowed some of the DCPIP to become reduced as this artificial electron acceptor likely accepted excessive electrons 


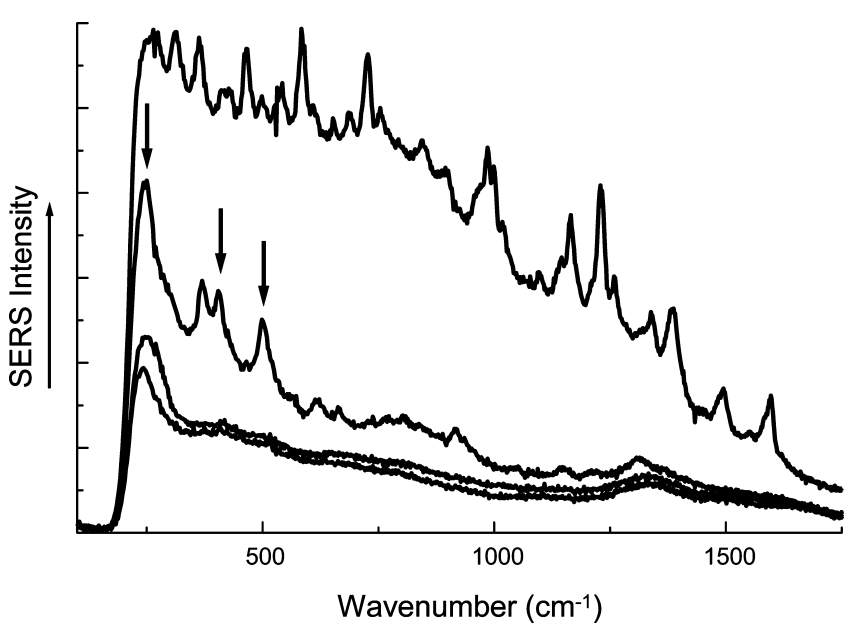

Figure 5. Representative raw Raman and SERS spectra, from top to bottom: Raman spectra of DCPIP in the solid state; followed by SERS spectra collected after 5 min aggregation at $\mathrm{pH} \mathrm{7.2,} \mathrm{5.5,} \mathrm{and} \mathrm{2.2,}$ respectively. The three vibrational bands from SERS that were used for kinetic analysis are indicated by arrows and these are at 243, 404, and $498 \mathrm{~cm}^{-1}$.

generated from the electron transport chain; the slope of this line was identical to the reaction mixture with $0.3 \mathrm{~mL}$ of mitochrondia preparation, which suggests that the concentration of mitochondria in this preparation was too low. By contrast, when the volume of mitochondria preparation was increased to 0.6 (upward pointing triangles) and $0.9 \mathrm{~mL}$ (circles) the slope decreased further showing that the enzyme reaction was faster as more enzyme was used. From these experiments we decided that as the enzyme reaction occurred fastest with $0.9 \mathrm{~mL}$ of mitochondria preparation this was to be used for the SERS experiments.

3.2. SERS Optimization. The Raman spectrum of powdered samples of DCPIP collected with an integration time of $10 \mathrm{~s}$ using full laser power $(300 \mathrm{~mW})$ is shown in Figure 5 and this information-rich spectrum contains plenty of molecular vibrations. However, while solution state spectra of DCPIP could also be collected at high concentrations, the signal was very low at $3.3 \times 10^{-5} \mathrm{~mol} \mathrm{dm}^{-3}$ (data not shown) which was the concentration used in the enzyme reaction (vide supra); therefore, without very long collection times, SERS would need to be employed.

One advantage of SERS is that intense Raman signals enable analyte concentrations well below monolayer coverage of the (gold) sol to be readily examined. Since aggregation is essential for obtaining enhanced intensities, control of the aggregation process has to be achieved if SERS is to be used. Based on previous studies ${ }^{8}$ we decided to use poly(L-lysine), which contains both positive and negative charged groups as well as $\mathrm{HCl}$ (which maintains an acidic environment), as this combination provides a less dynamic and thus more controllable aggregation procedure. ${ }^{21}$

For optimization of the SERS signal a dilute solution of the dye $\left(150 \mu \mathrm{L}\right.$; resulting in a final concentration of $3.3 \times 10^{-5}$ mol $\left.\mathrm{dm}^{-3}\right)$ was added to an aliquot of gold sol $(1 \mathrm{~mL})$ diluted with $1 \mathrm{~mL}$ of $\mathrm{H}_{2} \mathrm{O}$ to which was added $150 \mu \mathrm{L}$ of poly(L-lysine) $(0.01 \%)$ and either $\mathrm{HCl}$ or $\mathrm{KOH}$ to achieve the optimum solution $\mathrm{pH}$. After this induction of colloid aggregation with poly(Llysine), subsequent collection of SERS occurred after 5 min. Unfortunately, the spectra collected did not reveal vibrational bands attributed to DCPIP regardless of whether we increased further aggregation time or investigating other $\mathrm{pH}$ conditions.
It therefore appeared that the addition of poly(L-lysine) prevented collection of SERS spectra.

A subsequent set of experiments in the absence of poly $(\mathrm{L}-$ lysine) were conducted as described above. The $\mathrm{pH}$ conditions that were tested included $\mathrm{pH} 7.2,5.5$, and 2.2 and the resultant SERS spectra of $3.3 \times 10^{-5} \mathrm{~mol} \mathrm{dm}{ }^{-3}$ DCPIP collected with an integration time of $10 \mathrm{~s}$ using full laser power $(300 \mathrm{~mW})$ are shown in Figure 5. It can be seen in this figure that the best SERS spectrum (in terms of vibrational features) was when the $\mathrm{pH}$ of the aggregating reaction was conducted at $\mathrm{pH} 7.2$, with very few discernible features obtained from DCPIP under acidic conditions other than gold sol band at $\sim 250 \mathrm{~cm}^{-1}$.

3.3. Monitoring Succinate Dehydrogenase Activity Using SERS. Despite the above $\mathrm{pH}$ optimization, for monitoring the reduction of DCPIP by electron transfer the SERS aggregation was still assessed at the three different $\mathrm{pH}$ conditions as the dye could exist in either the protonated or deprotonated forms (see Figure 1 for the structure of DCPIP).

For SERS analysis at $\mathrm{pH} 5.5$ (the natural $\mathrm{pH}$ of the aggregating reaction) no vibrational bands could be seen, with the exception of the sol band at $238 \mathrm{~cm}^{-1}$ (Figure 6A) and it was therefore not surprising that no enzyme activity could be observed by plotting the intensity of this band against time at this $\mathrm{pH}$ (Figure 6B). When the experiment was repeated and the $\mathrm{pH}$ adjusted to 2.2 (Figure 6C) a direct correlation could be seen between the data acquired using UV/vis (Figure 5) and SERS with the disadvantage that only the sol vibrational band at $257 \mathrm{~cm}^{-1}$ was suitable for analysis of enzyme activity by SERS. A plot of SERS peak area versus time (Figure 6D) provided a good correlation coefficient $\left(R^{2}=0.983\right)$, but this is likely to be impractical as no spectral features from DCPIP were involved in this correlation.

By contrast, by adjusting the $\mathrm{pH}$ to 7.2 the most marked SERS effect was observed which resulted in strong vibrational bands (Figure 6E) where the bands at 243, 404, and $498 \mathrm{~cm}^{-1}$ could be used for quantitative analysis (Figure 6F) providing good correlation coefficients of $0.952,0.976$ and 0.939 , respectively. In addition, while these fits are calculated from linear regression it is clear from Figure 6F that as expected the enzyme kinetics measured are not really linear and are decaying as a function of time as the succinate substrate is being converted into the product fumarate (Figure 2).

An interesting physical chemistry observation relates to the quantitative decrease in the peak areas of the SERS vibrational bands vs time when comparison is made of the two experiments conducted at acid and neutral $\mathrm{pH}(\mathrm{pH} 2.2$ and 7.2) see Figure 6 , panels D and F. These data potentially suggest that two forms of the dye, protonated and deprotonated, are present, and this is feasible when the structure of DCPIP is inspected (see Figure 1). It can be hypothesized that the decrease in intensity (peak area) of the "sol" bands, as a function of time, for SERS experiments conducted at $\mathrm{pH} 2.2$ and 7.2 (viz., 257 and 243 $\mathrm{cm}^{-1}$, respectively) may be due to the packing effects of either competing species of DCPIP or artifacts of enzyme activity competing for the surface sites on gold. Packing effects (excess of monolayer coverage) on the surface would almost certainly decrease the signal intensity and at the start of the enzymatic reaction the DCPIP concentration is $3.3 \times 10^{-5} \mathrm{~mol} \mathrm{dm}^{-3}$. Successive layers of molecules on the surface of the nanoparticles would most likely be a contributory factor and appear to be the logical reason for the results observed; that is, an excess of monolayer coverage is being observed as enzyme activity proceeds. The effect of packing effects when collecting SERS spectra have been reported and this is a similar phenomenon 

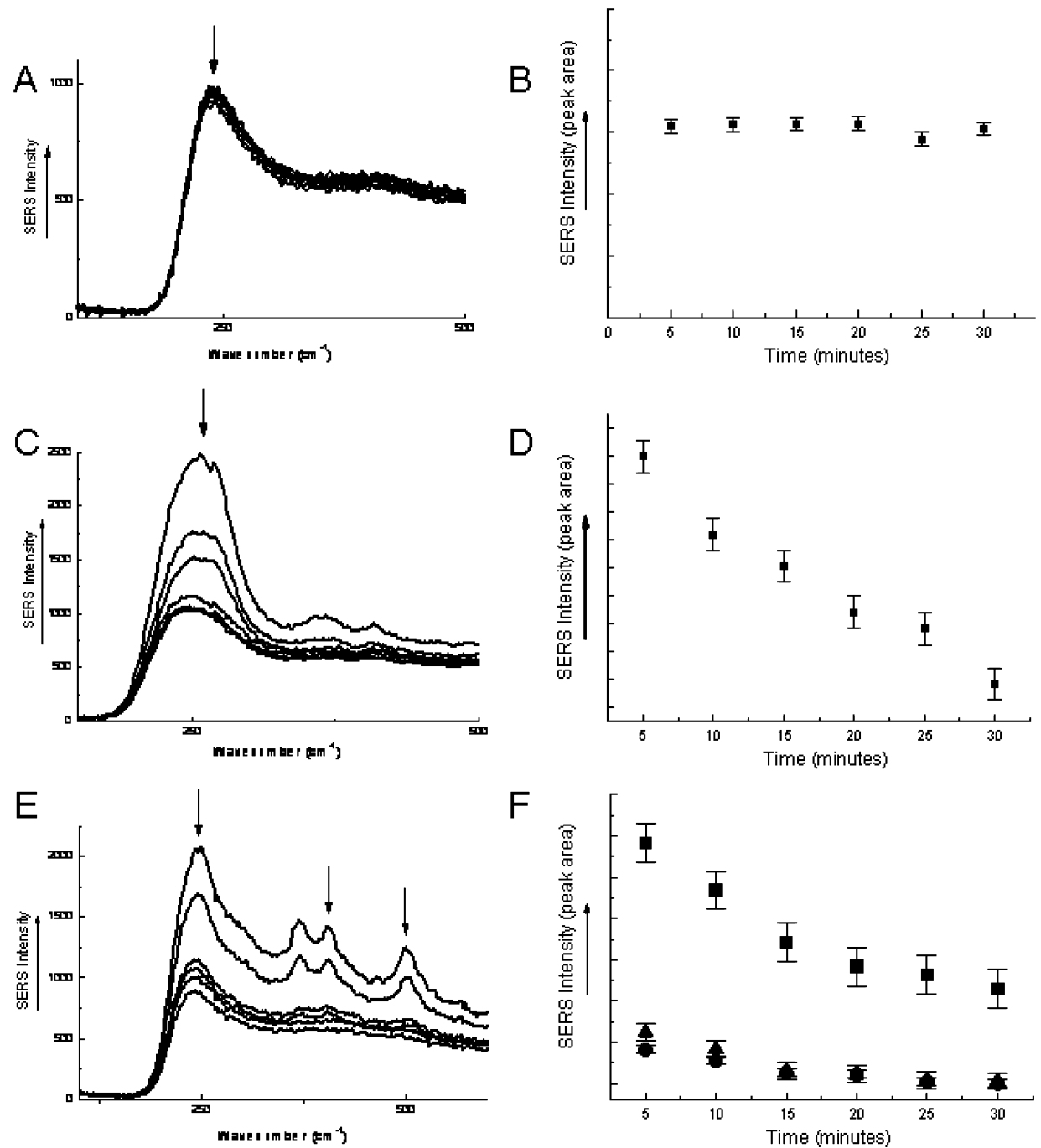

Figure 6. SERS spectra of the succinate dehydrogenase enzyme reaction. Aggregation was performed without poly(L-lysine) at (A) pH 5.5, (C) 2.2 , and (E) 7.2, from top to bottom the spectra shown were collected after 5, 10, 15, 20, 25, and 30 min of enzyme reaction. Plots of log 10 peak area versus time for the vibrational bands at (B) $238 \mathrm{~cm}^{-1}$ for measurements conducted at $\mathrm{pH} 5.5$, (D) $257 \mathrm{~cm}^{-1}\left(R^{2}=0.983\right)$ for SERS at pH 2.2 , and (F) for readings at $\mathrm{pH} 7.2$ for bands 243,404 , and $498 \mathrm{~cm}^{-1}\left(R^{2}=0.952,0.976\right.$, and 0.939 , respectively); error bars show standard deviations from the three replicate measurements.

that was observed in a separate study were it was shown that an excess of monolayer coverage led to a proportional decrease in signal intensity. ${ }^{22}$ Close inspection of the two sol bands for pH 2.2 (Figure 6D) and 7.2 (Figure 6F) show that the reduction of this band at $\mathrm{pH} 2.2$ is linear, which would support the above packing hypothesis. By contrast, at $\mathrm{pH} 7.2$ the decrease is nonlinear which shows that packing is not occurring and follows the classic enzyme kinetic reactions; due to depletion of substrate as detailed above. An observation that is also observed during the conversion of DCPIP from its oxidized to reduced forms for the other two peaks at 404 and $498 \mathrm{~cm}^{-1}$ (Figure 6F). These bands certainly arise from the DCPIP molecule itself and are possibly from $\mathrm{C}-\mathrm{Cl}$ stretches $\left(498 \mathrm{~cm}^{-1}\right)$ and skeletal vibrations $\left(404 \mathrm{~cm}^{-1}\right),{ }^{23}$ and the additional small intensity band at ca. 800 $\mathrm{cm}^{-1}$ (Figure 5) originates from ring breathing ${ }^{23}$ further highlighting that the reporter molecule itself is being measured.

\section{Concluding Remarks}

The main objective of this work was to monitor a metabolic pathway utilizing SERS as the reporting tool. For the first time a widely studied pathway, the kinetics of succinate dehydrogenase activity, is reported employing Raman spectroscopy to monitor this enzyme activity using a SERS reporter dye as a model system probe. The data reported clearly shows a direct correlation between acquisition of SERS spectra at specific time intervals and that obtained using classical spectrophotometric techniques. Very good correlations were observed for quantitative analysis as the reaction proceeded and the SERS aggregation protocol developed was $\mathrm{pH}$ dependent. In the future we plan to investigate the multiplexing of SERS where different reporter molecules are used which are "tuned" into different metabolic processes, as detailed for DNA sequence detection by Graham, Faulds, and colleagues. ${ }^{24-26}$ In conclusion, we believe that SERS has considerable potential for being applied to the analysis of metabolic processes, and to our knowledge this is the first study of a metabolic pathway that has been monitored by SERS.

Acknowledgment. K.H. thanks Stiefel Laboratories (U.K.) Ltd. for her studentship and I.T.S. and R.G. are very thankful to UK BBSRC for funding.

\section{References and Notes}

(1) Reichmann, H.; Wildenauer, D. Histochemistry 1991, 96, 251.

(2) Peterson, J.; Vibat, C.; Gennis, R. B. FEBS Lett. 1994, 355, 155. 

675.

(3) Trumpower, B. L.; Gennis, R. B. Annu. Rev. Biochem. 1994, 63,

(4) Dautry, C.; Conde, F.; Brouillet, E.; Mittoux, V.; Beal, M. F.; Bloch, G.; Hantraye, P. Neurobiol. Disease 1999, 6, 259.

(5) Nelson, D. L.; Cox, M. M. Lehninger: Principles of Biochemistry; W. H. Freeman: London, 2008.

(6) Ferraro, J. R.; Nakamoto, K. Introductory Raman Spectroscopy; Academic Press: London, 1994.

(7) Moskovits, M. Rev. Mod. Phys. 1985, 57, 783.

(8) Shadi, I. T.; Cheung, W.; Goodacre, R. Anal. Bioanal. Chem. 2009, 394,1833

(9) Kneipp, K.; Kneipp, H. ; Tzkan, I.; Dasari, R. R.; Feld, M. S.; Dresselhaus, M. S. Nonlinear Raman probe of single molecules attached to colloidal silver and gold clusters. In Optical Properties of Nanostructured Random Media; Springer-Verlag: Berlin, 2002; Vol. 82, pp 227.

(10) Moskovits, M.; Tay, L. L.; Yang, J.; Haslett, T. SERS and the single molecule. Opt. Prop. Nanostruct. Random Media 2002, 82, 215.

(11) Bailo, E.; Deckert, V. Chem. Soc. Rev. 2008, 37, 921.

(12) Graham, D.; Faulds, K. Chem. Soc. Rev. 2008, 37, 1042.

(13) Jarvis, R. M.; Goodacre, R. Chem. Soc. Rev. 2008, 37, 931.

(14) Qian, X.-M.; Nie, S. M. Chem. Soc. Rev. 2008, 37, 912.

(15) Shadi, I. T.; Chowdhry, B. Z.; Snowden, M. J.; Withnall, R. Chem. Commun. 2004, 24, 1436.

(16) Moore, B. D.; Stevenson, L.; Watt, A.; Flitsch, S.; Turner, N. J.; Cassidy, C.; Graham, D. Nat. Biotechnol. 2004, 22, 1133.

(17) Ingram, A.; Byers, L.; Faulds, K.; Moore, B. D.; Graham, D. J. Am. Chem. Soc. 2008, 130, 11846.

(18) Chaudry, M. A.; Amin, S.; Malik, M. T. Sep. Sci. Technol. 1996, 31,1309 .

(19) Whitney, A. V.; Van Duyne, R. P.; Casadio, F. J. Raman Spectrosc. 2006, 37, 993.

(20) Shadi, I. T.; Chowdhry, B. Z.; Snowden, M. J.; Withnall, R. Spectrochim. Acta A 2003, 59, 2213.

(21) Sirlin, C.; Burgard, M.; Leroy, M. J. F.; Prevost, M. J. Membr. Sci. 1990, 54, 299.

(22) Shadi, I. T.; Chowdhry, B. Z.; Snowden, M. J.; Withnall, R. Anal. Chim. Acta 2001, 450, 115.

(23) Degen, I. A. Tables of chracteristic group frequencies for the interpretation of infrared and Raman spectra; Acolyte Publications: Harrow, U.K., 1997.

(24) Graham, D.; Mallinder, B. J.; Whitcombe, D.; Watson, N. D.; Smith, W. E. Anal. Chem. 2002, 74, 1069.

(25) Faulds, K.; McKenzie, F.; Smith, W. E.; Graham, D. Angew. Chem. 2007, 46, 1829.

(26) MacAskill, A.; Crawford, D.; Graham, D.; Faulds, K. Anal. Chem. 2009, 81, 8134 .

JP908950X 